\title{
Neutrophil Extracellular Traps and Interleukin 17 in Ankylosing Spondylitis
}

\author{
Charalampos Papagoras ${ }^{1,2}$ (D), Akrivi Chrysanthopoulou², Alexandros Mitsios², Victoria Tsironidou², \\ Konstantinos Ritis ${ }^{1,2}$
}

${ }^{1}$ First Department of Internal Medicine, University Hospital of Alexandroupolis, Democritus University of Thrace, Alexandroupolis, Greece, '2Laboratory of Molecular Hematology, Democritus University of Thrace, Alexandroupolis, Greece

\section{ABSTRACT}

Ankylosing spondylitis (AS) is a chronic inflammatory disease traditionally regarded as mediated by $T$ lymphocytes. Recent progress has identified that cells of innate immunity are also important for the processes of inflammation and new bone formation, a hallmark of AS. Moreover, interleukin-17 (IL17 ) is a cytokine implicated in both processes. Neutrophils are increasingly recognized as mediators of autoinflammatory and autoimmune diseases through several mechanisms, one being the release of neutrophil extracellular traps (NETs). NETs are equipped with an array of bioactive molecules, such as IL-1 1 or IL-17. It appears that the molecules expressed over NETs vary across different disorders, reflecting diverse pathophysiologic mechanisms. As few studies have investigated the role of neutrophils in AS, the purpose of this research protocol is to study whether neutrophils from AS patients are more likely to form NETs, whether IL-17 and IL-1 $\beta$ are expressed over those NETs and if NETs affect new bone formation.

Mediterr J Rheumatol 2021;32(2):182-5

https://doi.org/10.31138/mjr.32.2.182

Article Submitted: 30 Jan 2020; Revised Form: 14 Mar 2021; Article Accepted: 30 Mar 2021; Available Online: 30 Jun 2021

Keywords: Ankylosing spondylitis, neutrophil extracellular traps, interleukin 17

\section{INTRODUCTION}

Ankylosing spondylitis (AS) is a chronic systemic inflammatory disease affecting both musculoskeletal and extraskeletal structures, such as the intestine, the skin, and the uvea. ${ }^{1}$ The musculoskeletal manifestations characteristically involve the spine, which is inflamed typically at the vertebral

\section{Corresponding Author:}

Charalampos Papagoras

First Department of Internal Medicine,

University Hospital of Alexandroupolis

Dragana, 68131, Alexandroupolis,

Greece.

Tel.: +30 6973690556

Fax: +302551030378

E-mail: cpapagor@med.duth.gr corners, as well as the apophyseal joints. The inflammation results in bone erosion ultimately followed by an aberrant reparative process, whereby neoosteogenesis, syndesmophyte formation, and bony ankylosis take place. $^{2}$ The fundamental pathogenetic process is considered to be enthesitis, ie, the inflammation of the attachment site to the bone of force-conveying fibrous structures, such as tendons and ligaments, the most typical being the enthesis of Achilles tendon. ${ }^{1}$

Due to the strong association between AS and the HLA B27 molecule, which, by operating as a major histocompatibility class II molecule, participates in the cognate activation of CD8+ positive T cells, AS was initially considered as an autoimmune disease driven by the inadvertent recognition of auto- or allo-antigens. However, evidence for the existence of such (auto)antigens is still poor. In contrast, alternative theories have suggested that HLA B27 may induce the activation of the immune system through non-cognate mechanisms, such as by forming aberrant structures, eg, misfolded or homodimeric complexes. ${ }^{3-5}$ Indeed, innate cells, such 
as innate lymphoid T cells (ILC), natural killer (NK) cells, CD14+ myeloid cells, and monocytes seem to play an important role in AS pathogenesis. Those cells mediate their effects through the secretion of cytokines, such as IL- 23, IL-17, IL-1ß, IL-22 and tumour necrosis factor-a (TNFa). ${ }^{6-10}$

Neutrophil is a cell type of key importance for the innate immunity: it is capable of sensing danger signals, reacting swiftly against pathogens and delivering signals to other cell types during the inflammatory process. Those functions are achieved through receptors, such as tolllike receptors (TLR), phagocytosis, degranulation and release of bioactive substances, such as enzymes, and, finally through a particular type of cell death, whereby the cell releases its chromatin in the form of a mesh, called neutrophil extracellular traps (NETs). ${ }^{11}$ Over NETs molecules like IL-1 $\beta, I L-17$, tissue factor or the antimicrobial protein LL-37 are often detected, which remain biologically active even following neutrophil death. ${ }^{12-19}$ NETs are involved in the pathogenesis of autoinflammatory diseases, like familiar Mediterranean fever (FMF) or adult-onset Still's disease (AOSD). During an FMF attack, neutrophils release NETs carrying IL-1 $\beta$, which further sustains inflammation. ${ }^{12-13}$ Besides, IL-1 $\beta$ blockade with anakinra or canakinumab are to date the most effective treatment of FMF, while similar observations have been published for AOSD as well. ${ }^{14-15}$ Even in classic autoimmune disorders, like systemic lupus erythematosus and ANCA-associated vasculitides, NETs appear to boost the autoimmune process by providing autoantigens and acting as a prothrombotic and proinflammatory machinery, exposing tissue factor, pro-inflammatory cytokines or modifying the function of other immune cells, such as dendritic cells, or non-immune cells, such as fibroblasts. ${ }^{18-22}$ In conclusion, NETosis possibly represents a generic mechanism of final neutrophilic reaction, which may vary in its details depending on the particular disorder. That is, a different set of molecules decorate NETs in each condition reflecting the neutrophil transcriptional process as it had been configured by the cell's microenvironment before NETosis occurred. ${ }^{23}$

Much less is known about neutrophils and NETs in the Spondyloarthritides (SpA). In the inflammatory bowel disease (IBD), a condition in which axial SpA is clinically manifest in almost 10\% of patients, neutrophils show diverse responses as far as NET formation is concerned. In the case of ulcerative colitis IL-1 $\beta$ bearing NETs are formed, while in Crohn's disease no NETosis is observed. ${ }^{24}$

The peripheral arthritis of SpA is characterized by the presence of IL-17 in the synovial fluid and the synovium itself, in which it is mainly expressed within mast cells and neutrophils, but not T cells. ${ }^{25}$ Notably, IL-17-containing neutrophils have been identified in vertebral biopsies of AS patients, representing the major IL-17-expressing cell type. ${ }^{26}$ On the other hand, the role of IL-17 in AS has gained much interest in the recent years. While it was initially thought to be produced by a particular type of $\mathrm{T}$ helper cells ( $\mathrm{TH} 17$ ), nowadays the role of IL-17-expressing cell types of innate immunity is gradually uncovered and includes type 3 innate lymphoid cells (ILC3), $\gamma / \delta T$ cells and NK cells. ${ }^{27}$

Moreover, IL-17 appears to be a cytokine involved not only in inflammation and bone erosion through RANKL upregulation, but also in new bone formation. ${ }^{27}$ However, the signals eliciting $\mathrm{L}-17$ release in the axial skeleton from the various cell types, as well as the sequence of events leading from IL-17 expression up to new bone formation, still remain unclear. The aim of the current protocol is to investigate the role of neutrophils in AS, particularly whether they form NETs, whether those NETs carry IL-17 or other bioactive molecules, as well as their biological effects on both aspects of AS, inflammation and bone metabolism.

\section{MATERIALS AND METHODS}

This is a prospective study that will be performed at the University Hospital of Alexandroupolis. Patients with AS, as well as non-AS control volunteers will be asked to participate in the study after giving their written informed consent. The study protocol has been approved by the Ethics Committee of the University Hospital of Alexandroupolis and the study will conform with the tenets of the Declaration of Helsinki.

Peripheral blood ( 20ml) will be collected from patients with active AS, patients with AS in remission/low disease activity and healthy donors. The estimated number of participants is 10 for either patient group and 20 for controls. Peripheral neutrophils will be isolated for immediate functional assays or stored for subsequent experiments. Serum will also be collected. For the bone formation experiments, part of bone marrow aspirates drawn for diagnostic purposes from patients being evaluated for anaemia (in the absence of neoplasia or systemic inflammation) will also be collected in order to obtain mesenchymal stem cells (MSCs)

The following clinical variables of AS patients will be recorded: year of birth, sex, height, weight, year of AS symptom onset, year of AS diagnosis, presence of peripheral arthritis, enthesitis, dactylitis, psoriasis, IBD, uveitis, carriage of HLA B27, presence of spinal syndesmophytes, treatment (non-steroidal anti-inflammatory drugs, synthetic disease-modifying anti-rheumatic drugs, glucocorticoids, biologic agents), measures of disease activity (erythrocyte sedimentation rate, C-reactive protein, BASDAI, ASDAS, patient's global evaluation of disease activity on a visual analogue scale). Levels of disease activity will be characterised according to currently accepted ASDAS cut-offs. 
Presence of NETS in AS

As an initial step to show whether NETs are involved in AS pathogenesis, neutrophils from patients with active AS will be cultured for 3 hours and subsequently stained with markers of neutrophils (neutrophil elastase, NE) and NET formation (citrullinated histone-3, citH3). DNA will be stained with DAPI. Observation will be performed with immunofluorescence microscopy.

For quantification of NET release, AS patient neutrophils will be cultured in appropriate medium for 4 hours and then NET structures will be isolated with intense shaking. MPO/DNA complex ELISA will subsequently be performed, which expresses NET release in a semi-quantitative manner. MPO/DNA complex ELISA will also be performed directly on the serum of AS patients. Healthy donor neutrophils and serum will be used as control in all the above experiments.

\section{Protein content of AS NETS}

The presence of IL-17A and IL-1 $\beta$ (a key cytokine in most autoinflammatory disorders) over AS-derived NETs will be examined with immunofluorescence using appropriate markers (staining for IL-17A/NE/DAPI or IL-1 $/$ /NE/DAPI) and isotype controls. To verify the presence of IL-17 or IL-1 $\beta$, AS neutrophils will be allowed to form NETs in culture and then NET proteins will be collected following NET digestion with a DNase-I. Interleukin-17 and IL-1 $\beta$ protein will be expressed semiquantitavely with immunoblotting, while ELISA will be performed to quantify IL-17 and IL-1 $\beta$ over NETs. The expression of the IL-17A gene in AS neutrophils will be investigated by quantifying IL17A mRNA using qPCR. The above experiments will be controlled using healthy donor neutrophils.

\section{Ability of AS inflammatory microenvironment to induce NETS}

Healthy donor neutrophils will be incubated in the presence of serum from AS patients or healthy donors and their ability to form NETs will be examined directly with immunofluorescence, as well as with MPO/DNA complex ELISA applied on NET structures. Further, the presence of IL-17A and IL-1 $1 \beta$ over those NETs will also be examined directly with immunofluorescence and with ELISA applied on NET structures. In order to examine the importance of AS inflammatory milieu, the above experiments will be repeated with the additional blockade of the effects of cytokines, particularly IL-1, IL-23 and TNFa.

Effect of the AS inflammatory microenvironment or NETS on the differentiation of bone marrow mesenchymal stem cells

The bone marrow mesenchymal stem cells (MSCs) are multipotent cells, which, depending on their microenvironment, may differentiate towards osteoblasts, chondrocytes or adipocytes. We will examine whether AS microenvironment or neutrophils have any effect on the proliferation and differentiation of MSCs. For this purpose, MSCs isolated from bone marrow aspirates from non-AS patients will be cultured in the presence of AS-derived serum or AS-derived NETs. Healthy serum and NETs formed by healthy donor neutrophils treated with ionomycin (a generic NET inducer) will serve as controls. The differentiation of MSCs towards bone forming cells will be assessed with special stains (Alizarin Red, von Kossa) in inverted microscope, as well as quantifying mRNA expression of genetic markers with qPCR, such as alkaline phosphate, osteocalcin, distal-less homeobox protein 5 and runt-related transcription factor 2 .

\section{IMPORTANCE OF THE STUDY}

Ankylosing spondylitis is a chronic inflammatory disease, causing pain, limiting patient function due to spinal inflammation, and producing chronic disability due to bony ankylosis. Despite recent progress, the core question on the sequence of events leading from inflammation to new bone formation remains unanswered. Although modern biological therapies are effective in suppressing inflammation, high response levels are achieved by less than a half of patients. ${ }^{28}$ Moreover, no treatment has been convincingly proved to halt structural damage so far. ${ }^{29}$ Given that the disease affects people during their most productive age, there is a great need to clarify the mechanisms of spinal inflammation and ankylosis, in order to optimize treatments and identify new therapeutic targets. There is plenty of evidence that in the pathophysiology of AS-related inflammation innate immunity mechanisms are at least as important as acquired immunity. Studies in other diseases have shown that neutrophils may express IL-17 and that, through NET formation, it may play a role in the initiation and prolongation of inflammation, but also in the modification of the function of non-immune cells in its vicinity. This study will be the first systemic investigation of neutrophils in AS aiming at answering several research questions:

- Whether neutrophils in AS show increased NET formation

- Whether NET formation parallels disease activity

- Whether AS-derived NETs carry IL-17 and/or IL-1 $\beta$

- The factors affecting NET formation in AS

- Whether NETs affect the phenotype of MSCs and, particularly, if they promote differentiation toward bone forming cells

\section{FUNDING}

The study is supported by a Research grant from the Hellenic Rheumatology

Society \& Professional Association of Rheumatologists (Decision Number 864/23-12-2019). 


\section{CONFLICT OF INTEREST}

The authors declare no conflict of interest.

\section{REFERENCES}

1. Rudwaleit M, Landewé R, Sieper J. Ankylosing spondylitis and axial spondyloarthritis. N Engl J Med 2016;375:1302-3.

2. Hermann KG, Baraliakos $X$, van der Heijde DM, Jurik AG, Landewé $\mathrm{R}$, Marzo-Ortega $\mathrm{H}$, et al; Assessment in SpondyloArthritis international Society (ASAS). Descriptions of spinal MRI lesions and definition of a positive MRI of the spine in axial spondyloarthritis: a consensual approach by the ASAS/OMERACT MRI study group. Ann Rheum Dis 2012;71:1278-88.

3. Chatzikyriakidou $A$, Voulgari $P V$, Drosos $A A$. What is the role of HLA-B27 in spondyloarthropathies? Autoimmun Rev 2011;10:4648.

4. Guiliano DB, North H, Panayoitou E, Campbell EC, McHugh K, Cooke FG, et al. Polymorphisms in the F pocket of HLA-B27 subtypes strongly affect assembly, chaperone interactions, and heavy-chain misfolding. Arthritis Rheumatol 2017;69:610-21.

5. Wong-Baeza I, Ridley A, Shaw J, Hatano H, Rysnik O, McHugh K, et al. KIR3DL2 binds to HLA-B27 dimers and free $\mathrm{H}$ chains more strongly than other HLA class I and promotes the expansion of $\mathrm{T}$ cells in ankylosing spondylitis. J Immunol 2013;190:3216-24.

6. Chan AT, Kollnberger SD, Wedderburn LR, Bowness P. Expansion and enhanced survival of natural killer cells expressing the killer immunoglobulin-like receptor KIR3DL2 in spondylarthritis. Arthritis Rheum 2005:52:3586-95.

7. Sherlock JP, Joyce-Shaikh B, Turner SP, Chao CC, Sathe M, Grein J, et al. IL-23 induces spondyloarthropathy by acting on ROR- $y t+C D 3+C D 4-C D 8-$ entheseal resident T cells. Nat Med 2012;18:1069-76.

8. Bridgewood C, Watad A, Russell T, Palmer TM, Marzo-Ortega H, Khan $A$, et al. Identification of myeloid cells in the human enthesis as the main source of local IL-23 production. Ann Rheum Dis 2019;78:929-33.

9. Ciccia F, Guggino G, Rizzo A, Saieva L, Peralta S, Giardina A, et al. Type 3 innate lymphoid cells producing $\mathrm{IL}-17$ and $\mathrm{IL}-22$ are expanded in the gut, in the peripheral blood, synovial fluid and bone marrow of patients with ankylosing spondylitis. Ann Rheum Dis 2015;74:1739-47.

10. Ciccia F, Guggino G, Zeng M, Thomas R, Ranganathan V, Rahman A, et al. Proinflammatory CX3CR1+CD59+Tumor Necrosis Factor-Like Molecule 1A+Interleukin-23+ monocytes are expanded in patients with ankylosing spondylitis and modulate Innate Lymphoid Cell 3 immune functions. Arthritis Rheumatol 2018;70:2003-13.

11. Rosales C. Neutrophil: A cell with many roles in inflammation or several cell types? Front Physiol. 2018;9:113.

12. Apostolidou E, Skendros P, Kambas K, Mitroulis I, Konstantinidis T, Chrysanthopoulou A, et al. Neutrophil extracellular traps regulate $\mathrm{IL}-1 \beta$-mediated inflammation in familial Mediterranean fever. Ann Rheum Dis. 2016;75:269-77.

13. Skendros P, Chrysanthopoulou A, Rousset F, Kambas K, Arampatzioglou A, Mitsios A, et al. Regulated in development and DNA damage responses 1 (REDD1) links stress with IL-1 $\beta$-mediated familial Mediterranean fever attack through autophagy-driven neutrophil extracellular traps. J Allergy Clin Immunol 2017;140:137887.

14. Papagoras C, Chrysanthopoulou A, Mitsios A, Arampatzioglou A, Ritis K, Skendros P. Autophagy inhibition in adult-onset Still's disease: still more space for hydroxychloroquine? Clin Exp Rheumato 2017;35 Suppl 108(6):133-4.

15. Ahn MH, Han JH, Chwae YJ, Jung JY, Suh CH, Kwon JE, et al. Neutrophil extracellular traps may contribute to the pathogenesis in adult-onset Still's disease. J Rheumatol 2019;46:1560-9

16. Arampatzioglou A, Papazoglou D, Konstantinidis T, Chrysanthopoulou A, Mitsios A, Angelidou I, et al. Clarithromycin enhances the antibacterial activity and wound healing capacity in type 2 diabetes mellitus by increasing LL-37 load on neutrophil extracellular traps. Front Immunol 2018;9:2064.

17. Stakos DA, Kambas K, Konstantinidis T, Mitroulis I, Apostolidou E, Arelaki S, et al. Expression of functional tissue factor by neutrophil extracellular traps in culprit artery of acute myocardial infarction. Eur Heart J 2015:36:1405-14.

18. Kambas K, Chrysanthopoulou A, Vassilopoulos D, Apostolidou E, Skendros P, Girod A, et al. Tissue factor expression in neutrophil extracellular traps and neutrophil derived microparticles in antineutrophil cytoplasmic antibody associated vasculitis may promote thromboinflammation and the thrombophilic state associated with the disease. Ann Rheum Dis 2014;73:1854-63.

19. Frangou E, Chrysanthopoulou A, Mitsios A, Kambas K, Arelaki S, Angelidou I, et al. REDD1/autophagy pathway promotes thromboinflammation and fibrosis in human systemic lupus erythematosus (SLE) through NETs decorated with tissue factor (TF) and interleukin-17A (IL-17A). Ann Rheum Dis 2019;78:238-48.

20. van Dam LS, Kraaij T, Kamerling SWA, Bakker JA, Scherer UH, Rabelink TJ, et al. Intrinsically distinct role of neutrophil extracellular trap formation in antineutrophil cytoplasmic antibody-associated vasculitis compared to systemic lupus erythematosus. Arthritis Rheumatol 2019;71:2047-58.

21. Papadaki G, Kambas K, Choulaki C, Vlachou K, Drakos E, Bertsias $G$, et al. Neutrophil extracellular traps exacerbate Th1-mediated autoimmune responses in rheumatoid arthritis by promoting DC maturation. Eur J Immunol 2016;46:2542-2554.

22. Chrysanthopoulou A, Mitroulis I, Apostolidou E, Arelaki S, Mikroulis D, Konstantinidis, $T$ et al. Neutrophil extracellular traps promote differentiation and function of fibroblasts. J Pathol 2014;233:294307.

23. Mitsios A, Arampatzioglou A, Arelaki S, Mitroulis I, Ritis K. NETopathies? Unraveling the dark side of old diseases through neutrophils. Front Immunol 2017;7:678.

24. Angelidou I, Chrysanthopoulou A, Mitsios A, Arelaki S, Arampatzioglou A, Kambas K, et al. REDD1/Autophagy pathway is associated with neutrophil-driven IL-1 $\beta$ Inflammatory response in active ulcerative colitis. J Immunol 2018;200:3950-61.

25. Noordenbos T, Yeremenko N, Gofita I, van de Sande M, Tak PP, Caňete JD, et al. Interleukin-17-positive mast cells contribute to synovial inflammation in spondylarthritis. Arthritis Rheum 2012;64:99-109.

26. Appel H, Maier R, Wu P, Scheer R, Hempfing A, Kayser R, et al. Analysis of $\mathrm{IL}-17(+)$ cells in facet joints of patients with spondyloarthritis suggests that the innate immune pathway might be of greater relevance than the Th17-mediated adaptive immune response. Arthritis Res Ther 2011;13(3):R95.

27. McGonagle DG, Mclnnes IB, Kirkham BW, Sherlock J, Moots R. The role of IL-17A in axial spondyloarthritis and psoriatic arthritis: recent advances and controversies. Ann Rheum Dis 2019;78:1167-78.

28. Sepriano A, Regel A, van der Heijde D, Braun J, Baraliakos X, Landewé R, et al. Efficacy and safety of biological and targeted-synthetic DMARDs: a systematic literature review informing the 2016 update of the ASAS/EULAR recommendations for the management of axial spondyloarthritis. RMD Open 2017;3:e000396.

29. Aouad K, Ziade N, Baraliakos X. Structural progression in axial spondyloarthritis. Joint Bone Spine 2019 May 5. pii: S1297319X(19)30069-7. 